\title{
Dynamics and Mechanisms of Interfacial Photoinduced Electron Transfer Processes of Third Generation Photovoltaics and Photocatalysis
}

\author{
Christophe Bauera, Joël Teuscher ${ }^{b}$, Jan C. Brauer, Angela Punzi, Arianna Marchioro, Elham Ghadiri, \\ Jelissa De Jonghe, Mateusz Wielopolski, Natalie Banerji, and Jacques-E. Moser ${ }^{\star}$
}

\begin{abstract}
Photoinduced electron transfer (PET) across molecular/bulk interfaces has gained attention only recently and is still poorly understood. These interfaces offer an excellent case study, pertinent to a variety of photovoltaic systems, photo- and electrochemistry, molecular electronics, analytical detection, photography, and quantum confinement devices. They play in particular a key role in the emerging fields of third-generation photovoltaic energy converters and artificial photosynthetic systems aimed at the production of solar fuels, creating a need for a better understanding and theoretical treatment of the dynamics and mechanisms of interfacial PET processes. We aim to achieve a fundamental understanding of these phenomena by designing experiments that can be used to test and alter modern theory and computational modeling. One example illustrating recent investigations into the details of the ultrafast processes that form the basis for photoinduced charge separation at a molecular/bulk interface relevant to dye-sensitized solar cells is briefly presented here: Kinetics of interfacial PET and charge recombination processes were measured by fs and ns transient spectroscopy in a heterogeneous donor-bridge-acceptor (D-B-A) system, where $\mathrm{D}$ is a Ru"(terpyridyl- $\left.\mathrm{PO}_{3}\right)(\mathrm{NCS})_{3}$ complex, $\mathrm{B}$ an oligo-p-phenylene bridge, and A nanocrystalline $\mathrm{TiO}_{2}$. The forward $\mathrm{ET}$ reaction was found to be faster than vibrational relaxation of the vibronic excited state of the donor. Instead, the back ET occurred on the $\mu$ s time scale and involved fully thermalized species. The D-A distance dependence of the electron transfer rate was studied by varying the number of $p$-phenylene units contained in the bridge moiety. The remarkably low damping factor $\beta=0.16 \AA^{-1}$ observed for the ultrafast charge injection from the dye excited state into the conduction band of $\mathrm{TiO}_{2}$ is attributed to the coupling of electron tunneling with nonequilibrium vibrations redistributed on the bridge, giving rise to polaronic transport of charges from the donor ligand to the acceptor solid oxide surface.
\end{abstract}

Keywords: Femtochemistry · Molecular/bulk heterojunction · Photocatalysis · Photoinduced electron transfer . Third-generation solar cells

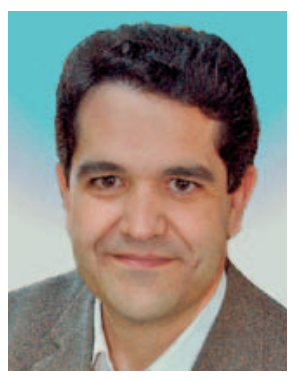

\section{Introduction}

The long-term goal of our research program is to contribute to the advancement of solar energy conversion science and technology through basic research. Currently, we focus our research on the preparation and characterization of photo-
${ }^{*}$ Correspondence: Prof. Dr J.-E. Moser Photochemical Dynamics Group Institute of Chemical Sciences and Engineering Ecole Polytechnique Fédérale de Lausanne EPFL SB ISIC GR-MO, Station 6 $\mathrm{CH}-1015$ Lausanne

E-mail: je.moser@epfl.ch

aPresent address: Gymnase Provence, $\mathrm{CH}-1007$ Lausanne

bPresent address: Condensed Matter Physics, Clarendon Laboratory, Department of Physics, University of Oxford, UK voltaic and photocatalytic systems, as well as on the understanding of the underlying processes. ${ }^{[1-5]}$ In this context, it is particularly interesting to elucidate the dynamics of fundamental processes (such as photoinduced electron transfer, charge transport, energy transfer and relaxation), which prevail in the nanomaterials and their interfaces. These processes are not only essential to the functioning of the studied system itself, but may also be relevant for many other materials and applications.

We utilize state-of-the-art time-resolved spectroscopic techniques to investigate these phenomena. Identification of reaction intermediates and quantification of the kinetics of photoinduced reactions are achieved by the application of a combination of various spectroscopic techniques. This includes femtosecond-, picosecond- and nanosecond pulsed laser excitation, coupled to various fast transient absorbance, diffuse reflectance, nonlinear second harmonic generation, and photoemission probing techniques. The timeresolution of current laser equipments allows for the monitoring of temporal domains extending over fifteen orders of magnitude, roughly from a few femtoseconds to seconds. Moreover we are able to employ all optical wavelengths in the UV, visible, NIR, and mid-IR spectral ranges. In addition, terahertz time-domain spectroscopy (THz-TDS) allows the dynamics of low frequency vibrations in molecules, solvents, solids, and supramolecular systems to be studied, as well as charge carrier mobility and transport mechanisms in nanomaterials. ${ }^{[4-7]}$

\section{Light-driven Charge Separation}

Photoinduced electron transfer (PET) has a great impact on modern chemistry and biology. PET processes have widespread application in semiconductor photocatalysis, ${ }^{[8]}$ imaging systems, such as silver halide photography, ${ }^{[9]}$ spectral sensitization, ${ }^{[10]}$ and xerography. ${ }^{[11]}$ Nature invokes electron transfer in a variety of enzymatic processes, such as oxidative 


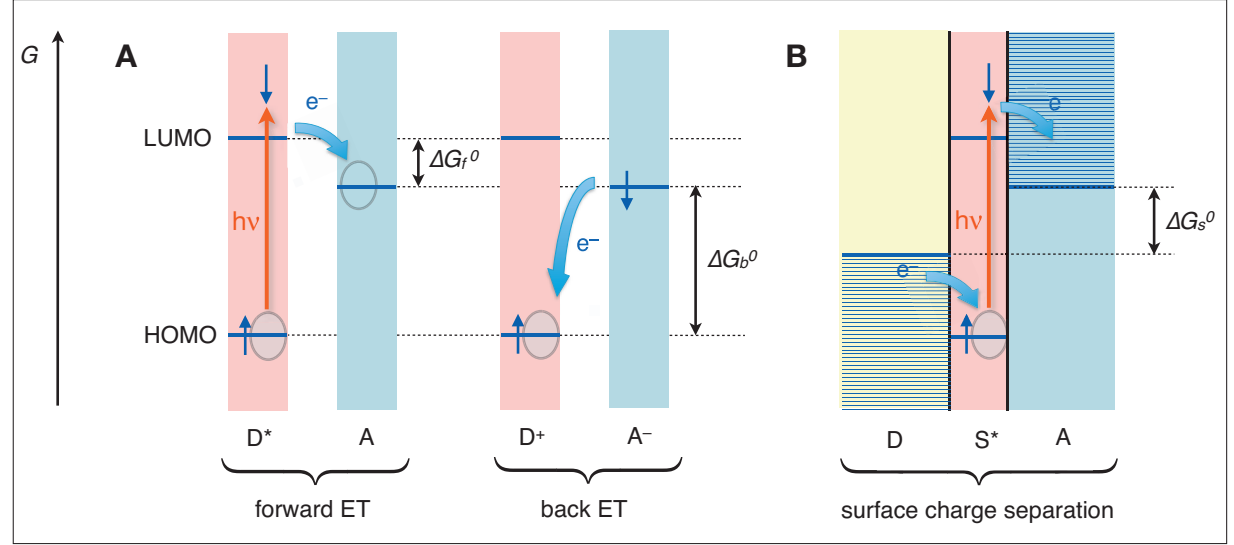

Fig. 1. (A) Energy scheme for photoinduced electron transfer from the electronic excited state $\left(D^{*}\right)$ of a donor species to an acceptor $(A)$ in solution yields the charge separated pair $\left[D^{+} \ldots A^{-}\right]$. Back electron transfer process is always thermodynamically spontaneous and leads quickly to the recombination of the charge separated pair. (B) Light-driven charge transfer at the surface between two materials forms a long-lived electron-hole pair, whose recombination is hampered by the interface.

phosphorylation, ${ }^{[12]}$ the DNA-photolyase reaction, ${ }^{[13]}$ and photosynthesis. ${ }^{[14]}$

In homogeneous media, PET involves the exchange of electrical charges between two discrete molecular states, which are typically in the solution phase. The primary step of the reaction consists of the conversion of the electronic excited state energy into chemical energy retained in the form of a redox geminate ion pair $\left(\mathrm{A}^{*}+\mathrm{D}\right.$ $\rightarrow\left[\mathrm{A}^{-} \ldots \mathrm{D}^{+}\right]$or $\left.\mathrm{A}+\mathrm{D}^{*} \rightarrow\left[\mathrm{A}^{-} \ldots \mathrm{D}^{+}\right]\right)$. In polar solvents, separation of the geminate pair occurs to form solvated ions in solution $\left(\left[\mathrm{A}^{-} \ldots \mathrm{D}^{+}\right] \rightarrow \mathrm{A}^{-}+\mathrm{D}^{+}\right)$. However, the quantum yield of product formation either from the reaction of the free ions, or of the geminate pair - is often low, due to the return electron transfer reaction $\left(\left[\mathrm{A}^{-} \ldots \mathrm{D}^{+}\right] \rightarrow \mathrm{A}+\mathrm{D}\right.$ or $\mathrm{A}^{-}+\mathrm{D}^{+} \rightarrow$ $\mathrm{A}+\mathrm{D})$. The latter process is an energywasting step that is always thermodynamically spontaneous and competes with the sustained charge separation and with other possible deactivation mechanisms involving further use of the charge-separated species (Fig. 1A).

Natural photosynthesis is the most fundamental bioenergetic process and serves as the best example of solar energy conversion. Besides being responsible for the evolution of the Earth's atmosphere, it is also the main route for providing free energy of the environment to the living world. Lightinduced charge separation is achieved through judicious spatial arrangement of the pigments and elements of the electron transport chain in the thylakoid membrane. Co-operative interaction between these components allows the electron transfer to proceed in a vectorial fashion.

One of the most important goals of chemistry over the past decades has been the construction and development of artificial molecular and supramolecular solar energy harvesting systems with the ability to absorb sunlight and convert it into useful and storable forms. Although strategies to design artificial photoconversion devices should not attempt to blindly imitate all the intricacies of natural photosynthesis, it is inconceivable to accomplish the challenging task of converting visible light into electrical work or chemical potential without suitable engineering on the molecular level. ${ }^{[15]}$

In all practical applications of PET reactions, charge separation has to be sufficiently long-lived for further redox reactions to take place or for charge carriers to be collected at electrical contacts. One way to achieve this goal is to operate charge transfer through a molecular/bulk interface. Charge injection from the photoexcited state of a molecule $\left(\mathrm{S}^{*}\right)$ adsorbed at a solid surface into an acceptor material (A) yields the oxidized species $\mathrm{S}^{+}$(Eqn. (1)), In turn, $\mathrm{S}^{+}$can abstract an electron from a donor (D) to regenerate the original absorber molecule S (Eqn. (2)). The same result can be obtained upon initial hole injection from the photoexcited molecule into the donor material (Eqn. (3)) and subsequent reaction of the reduced state product $\left(\mathrm{S}^{-}\right)$ with the acceptor (Eqn. (4)).

$$
\begin{aligned}
& \mathrm{S}^{*}+\mathrm{A} \rightarrow \mathrm{S}^{+}+\mathrm{A}\left(\mathrm{e}^{-}{ }_{\mathrm{cb}}\right) \\
& \mathrm{S}^{+}+\mathrm{D} \rightarrow \mathrm{S}+\mathrm{D}\left(\mathrm{h}^{+}{ }_{\mathrm{vb}}\right) \\
& \mathrm{S}^{*}+\mathrm{D} \rightarrow \mathrm{S}^{-}+\mathrm{D}\left(\mathrm{h}^{+}{ }_{\mathrm{vb}}\right) \\
& \mathrm{S}^{-}+\mathrm{A} \rightarrow \mathrm{S}+\mathrm{A}\left(\mathrm{e}^{-}{ }_{\mathrm{cb}}\right)
\end{aligned}
$$

The light-driven charge injection into the continua of electronic states that characterize the conduction band $(\mathrm{cb})$ and the valence band (vb) of acceptor and donor solids, respectively, is generally a fast process. Rapid delocalization of electrons and holes imposes an entropic barrier to their recombination. Local electric fields that may appear spontaneously at the D/A interface can also considerably retard back electron transfer by further separating opposite charges on both sides of the D/S/A interface (Fig. 1B).

During past decades, the movement of charge through individual molecules, as well as through solid-state structures, has been carefully investigated and successfully described theoretically. Due to the additional complexity imposed by the large number of electronic levels coupled to the reaction coordinate at surfaces, the heterogeneous charge transfer problem becomes much more complex at molecular/bulk interfaces and is still poorly understood. Nevertheless, a vast majority of successful applications involve photoredox processes and interfacial PET. Therefore, charge transport across interfaces remains the most important area of study in the budding field of molecular electronics. PET at molecular/bulk interfaces constitutes the major focus of research in the chemistry of photoelectrolysis, ${ }^{[16]}$ and photocatalysis. ${ }^{[8]}$ Molecular/bulk interfacial PET is also the primary step in many solar energy-conversion devices as it creates free charge carriers upon the absorption of a photon.

\section{Molecular Photovoltaic Systems}

The increased demand for renewable energy sources has prompted researchers to propose and test a great variety of novel photovoltaic designs, with respect to finding an effective alternative to silicon-based solar cells. Research and development in this area generally aim at higher efficiencies and lower costs per watt of electricity generated. Some in the solar cell industry identify different 'generations' of solar cell technology. Devices based on solidstate silicon $p$ - $n$ homojunctions are usually referred to as first-generation solar cells. Silicon is an indirect bandgap material with a low absorption coefficient $(\alpha=$ $5 \cdot 10^{2} \mathrm{~cm}^{-1}$ at $\left.1.5 \mathrm{eV}\right) .^{[17]}$ Thus, for efficient light harvesting a considerably thick layer of $c a .200 \mu \mathrm{m}$ is required. The semiconductor assumes two roles simultaneously: On the one hand, it harvests the incident sunlight and, on the other hand, it transports the charge carriers generated by light excitation. In order to operate at high efficiency, the photons have to be absorbed in the vicinity of the $p-n$ interface. Electronhole pairs produced at a distance from the junction must diffuse to the contact, where the local electrical field separates the charges (Fig. 2A). Defects in the transport material act as traps for the electrons and are responsible for charge recombination. To avoid charge carriers recombination during diffusion, the concentration of defects must be minimal. This imposes 

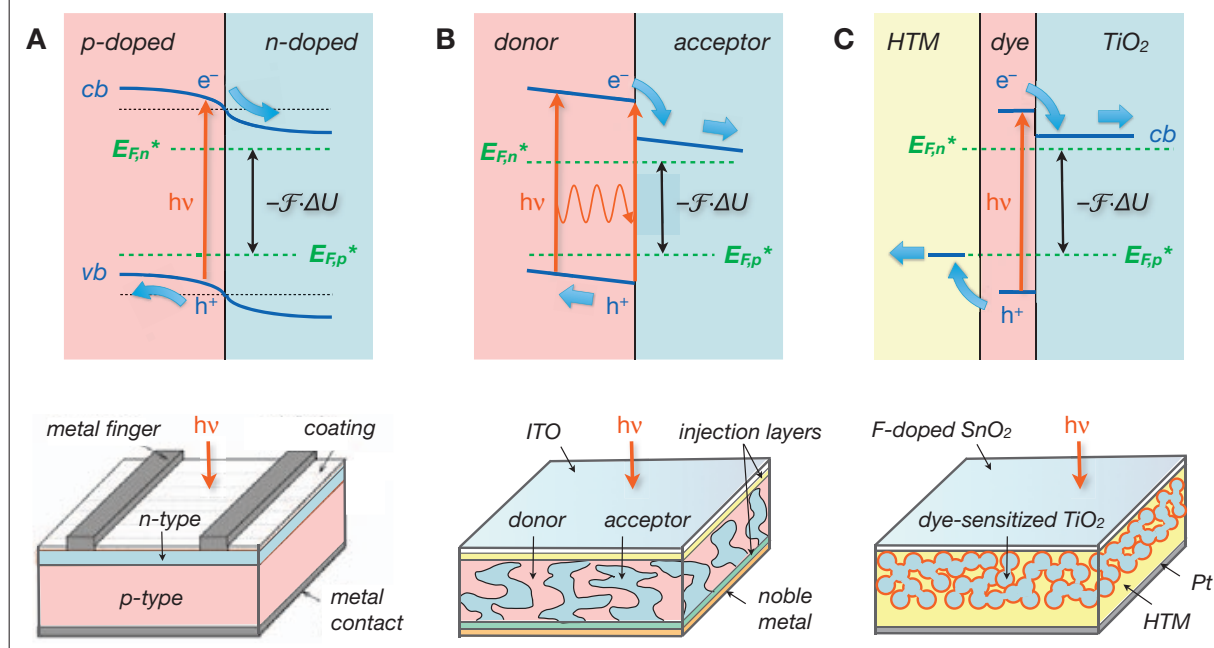

Fig. 2. Energetic scheme and structure principle of (A) a conventional $p-n$ semiconductor homojunction solar converter, (B) the donor-acceptor heterojunction of a solid-state organic photovoltaic device (OPV), and (C) a dye-sensitized solar cell (DSC). In the latter two cases, the distributed interface between the electron-conducting solid and the hole-transporting material (HTM) forms a bulk heterojunction.

severe requirements on the purity of the semiconductor material, rendering solidstate devices of the first generation type quite expensive.[17]

Second-generation solar cells are based on thin films of direct bandgap semiconductors, whose absorption coefficients are typically a thousand times larger than that of silicon. Cadmium telluride (CdTe, $\alpha=$ $5 \cdot 10^{5} \mathrm{~cm}^{-1}$ at $\left.1.5 \mathrm{eV}\right)^{[18]}$ and copper indium gallium selenide (CIGS) are two common materials used for outdoor photovoltaic solar power production. Thin-film technologies reduce the amount of material required for the production of a solar cell. Although this reduces material cost and energy payback time, it may also reduce energy conversion efficiency.

The third generation is somewhat ambiguous in the technologies that it encompasses, though generally it tends to include tandem/multi-junction cells, hot-carrier cells, dye-sensitized semiconductor solar cells (DSC), organic donor-acceptor photovoltaic devices (OPV), based either on conjugated polymer or small molecule absorbers, hybrid assemblies of inorganic and organic semiconductors, as well as solar cells employing quantum dots, fullerenes, carbon nanotubes and upconversion technologies. OPV and DSC, in particular, rely on molecular light absorbers and are in the focus of our research. These systems are made of low-cost materials and do not need elaborate equipment to manufacture. They are significantly less expensive than conventional $p$ - $n$ junction solar cells. Another advantage is that OPV and DSC devices can be easily engineered into flexible sheets with attractive designs. Hence, although their conversion efficiency is less than those of the best thin film cells, their price/performance ratio allows them to compete with older generation solar converters.

In a standard OPV device, ${ }^{[19]}$ the photoactive region generally consists of two materials (Fig. 2B). One of these serves as the light-harvesting electron donating material and the other as the electron accepting material. Photons impinging on the photoactive layer cause electronic excitations in the donor material and lead to the formation of excitons. Excitons are bound couples of a highly energetic electron and a positively charged electron vacancy or hole. To generate current, the exciton, i.e. the electron-hole pair, must migrate to the interface between the electron donor and acceptor materials. There it can be split into two separate mobile charges. From there the charges then diffuse to the respective electrodes - electrons are transported in the electron acceptor material to the anode, and holes in the electron donor material to the cathode.

One way to physically bring the acceptor and donor materials together is to deposit a very thin layer (thickness of typically of a few tens of nanometers) of the light-absorbing material onto a substrate of the corresponding donor or acceptor. This arrangement, known as a planar heterojunction, is generally used to fashion solar cells from small-molecule (nonpolymeric) organic compounds, which possess a large extinction coefficient. This simple planar design is not adapted to systems based on conjugated polymers, where several microns of the material are necessary to harvest the incident light. Furthermore, it should be considered that exciton diffusion to the interface (intermolecular energy transfer within the absorb- ing material) competes with electron-hole recombination and restricts the exciton diffusion length to a few nanometers only. To increase the interfacial area and decrease the distance that excitons have to travel to reach the interface, donor and acceptor components are blended in such a way that an interpenetrating bi-continuous network of junctions is formed. In such an arrangement, which is referred to as a bulk heterojunction (BHJ), the polymer and acceptor phases are intermingled on the nanometer scale (Fig. 2B).

Photovoltaic systems based on the sensitization of a wide bandgap semiconductor separate the functions of light absorption and carrier transport. ${ }^{[2,10]}$ Light harvesting is carried out by a dye-sensitizer molecule, which initiates electron transfer events leading to charge separation. A molecular dye coupled to nanocrystalline $\mathrm{TiO}_{2}$ is an excellent model for processes that occur in the above fields. This system represents one of the best-studied photovoltaic devices: the dye-sensitized nanocrystalline solar cell (DSC). These solar converters are based on transition-metal complexes or organic dye molecules that are adsorbed onto a highly porous nanocrystalline $\mathrm{TiO}_{2}$ layer. From the ground state, which is energetically located in the semiconductor band gap, the dye-sensitizer molecule is excited by visible light to an energetically higher electronic excited state, which is resonant with the $\mathrm{TiO}_{2}$ conduction band (cb) (Fig. $2 \mathrm{C})$. One electron is then injected into the semiconductor on an ultrafast timescale. In the following step, the oxidized dye species is reduced back to the initial chromophore state by electron transfer from a donor material filling the pores of the $\mathrm{TiO}_{2}$ mesoporous network. Redox couples in solution, redox-active ionic liquids, molecular liquids, or solid hole-transporting materials (HTM) can serve as donor materials. Finally, the generated electrons and holes (positive charges) travel through the $\mathrm{TiO}_{2}$ and the HTM interpenetrating phases to the anode and the cathode, respectively. The distributed HTM/molecular dye/inorganic semiconductor interface is another example of bulk heterojunction.

Convergence between the working principles behind OPV and DSC devices is reached in the case of organic solar cells based on small molecule absorbers and a HTM added. Moreover, hybrid organicinorganic bulk heterojunction systems also combine the concepts of DCS and OPV cells. In a hybrid organic-inorganic system a solid-state donor, which is capable of photoinduced electron injection into the conduction band of a metal oxide, forms an interpenetrating network with the pores of a nanocrystalline material. As well, the DSC principle gets quite close to that of an OPV cell when a solid-state donor capable 
of injecting electrons in the conduction band of a metal oxide upon light absorption is filling the pores of the nanocrystalline material, forming a hybrid organicinorganic bulk heterojunction system.

In nanodispersed semiconductors, no significant concentration gradient of charge carriers can establish within particles whose dimensions are smaller than the Debye length. The Debye length characterizes the thickness of the space-charge layer formed at the surface of bulk semiconducting materials. ${ }^{[2]}$ Rather than relying on an electric field to separate photogenerated electron-hole pairs, sustained light-induced charge separation in bulk heterojunction photovoltaic devices is based on the kinetic competition between energy- and electron transfer and charge transport processes. ${ }^{[3]}$ Understanding dynamics in photovoltaic and photocatalytic nanomaterials is, therefore, essential for improving efficiencies of existing devices and developing new concepts for solar energy conversion. Currently this notion is well accepted by the research community and an explosion of research efforts has been observed in this area in the last few years. This trend will continue in the near future as solar energy conversion research efforts experience steady growth.

\section{Ultrafast Charge Injection: Beyond Current Theories of ET}

In DSCs, ultrafast electron injection from a molecular excited state into the conduction band of a wide-bandgap semiconductor is key to the initial interfacial lightinduced charge separation. It is important in this context that electron injection has to compete efficiently with fast radiative and nonradiative deactivation pathways and quenching reactions. The dye cations produced as a consequence of charge injection have to be intercepted prior to their recombination with conduction band electrons. To a great extent, this charge transfer between the oxidized sensitizer at the surface and the hole transporting medium defines the photon-to-current conversion efficiency of the solar cell. In order to prevent indirect electron-hole recombination, both the percolation of electrons between the semiconductor nanoparticles and the transport of holes to the cathode within the pore network have to be sufficiently rapid.

Recent ultrafast studies have shown that PET from the singlet excited state of chemisorbed molecular sensitizers with a suitable redox potential into empty electronic states of oxide semiconductors to occur in the femtosecond to picosecond time domain. ${ }^{[3,20]}$ The time constant for ET found in these studies varies from a mere $6 \mathrm{fs}^{[21]}$ to hundreds of ps. ${ }^{[22,23]}$ Charge transfer times $\leq 25$ fs indicate that the reactions occur on the same time scale or even faster than nuclear motion associated with high-frequency intramolecular vibrations $\left(\bar{v} \geq 1600 \mathrm{~cm}^{-1}\right)$. In this case, PET processes do not involve a complete redistribution of vibrational excitation energy and thus cannot be described within the current Marcus-Levich-Jortner-Gerischer theory, which postulates vibration-mediated ET processes at thermal equilibrium. [21b,24] The notion that the electron is transferred to the solid well before vibrational relaxation of the photoexcited sensitizer has recently been confirmed in strong coupling cases by the observation of the dependence of ET kinetics upon the excitation photon energy, ${ }^{[25]}$ and by characterizing the oscillations due to vibrational wavepacket motion during heterogeneous charge transfer in the transient absorption signal.[21b,26]

The simplest kinetic model, which describes the charge injection as a non-adiabatic radiationless process, is derived from Fermi's golden rule. The rate constant for the reaction can then be expressed as the product of a Franck-Condon weighted density of states $(F C W D)$, which depends on the driving force $-\Delta G^{0}$, as well as the nuclear reorganization energy accompanying the electron transfer, and an electronic factor which is proportional to the electronic coupling matrix element squared $|H|^{2}$ :

$$
k_{e t}=\frac{2 \pi}{\hbar} \cdot|H|^{2} \cdot F C W D
$$

$F C W D$ is the integrated overlap of the reactant's and product's nuclear wavefunctions with equal energy. For a large number of accessible acceptor levels, the summation over all terms of this nuclear factor may be reduced to a pure density of final electronic states. ${ }^{[27]}$ In the non-adiabatic case, where the electronic coupling of the donor and acceptor is not very large (typically $\left.|H|<150 \mathrm{~cm}^{-1} \cong 0.7 \mathrm{k}_{\mathrm{B}} T\right), F C W D$ becomes a constant, which implies that the rate of the charge injection process is solely controlled by the electronic factor $|H|^{2}$. Obviously the role of the electronic coupling factor is of considerable interest. Its magnitude is determined by the separation distance and anchoring geometry of the sensitizer on the surface. In electron transfer reactions where the electron donor and acceptor are separated by a fixed distance $r$, Gamow's exponential relation (Eqn. (6) $)^{[28]}$ is used to estimate the changes in $|H|^{2}$ :

$$
\begin{aligned}
|H|^{2} & =|H|_{0}^{2} \cdot \exp \left[-\beta\left(r-r_{0}\right)\right] \Rightarrow \\
k_{e t} & =k_{e t}^{0} \cdot \exp \left[-\beta\left(r-r_{0}\right)\right]
\end{aligned}
$$

The damping factor $\beta$ is an exponential coefficient that describes the decay of the electronic wavefunction. The values for $\beta$ typically range from 0.2 to $2.5 \AA^{-1}$. Provided that other factors do not influence the electron transfer rate, Eqn. (6) can be used to estimate the rate constant $k_{e t}$ at a known separation distance.

\section{Ultrafast Charge Transfer through Oligo-( $p$-phenylene) Bridges: Effect of Non-equilibrium Vibrations}

The strong electronic coupling prevailing for an efficient sensitizer results from the anchoring of the dye molecule onto the semiconductor surface through a moiety carrying its lowest unoccupied molecular orbital (LUMO). This situation is clearly encountered in carboxylated $\mathrm{Ru}$ (II) polypyridyl complexes, ${ }^{[1]}$ and organic sensitizers, such as coumarin, [29] alizarin, ${ }^{[21,30]}$ and other D- $\pi$-A push-pull dyes, ${ }^{[10 b]}$ for example. The electronic coupling for PET can be diminished deliberately by increasing the distance separating the LUMO of the dye from the surface of the semiconductor material. This can be achieved, for instance, by inserting bridge units between the chromophore and the anchoring group of the dye molecule.

Fig. 3 shows the molecular structures of three dye-sensitizers used to investigate the distance dependence of ET rates. [31] Anchoring of these molecules onto the surface of titanium dioxide occurs via non-conjugated phosphonate groups. The use of spacer bridges based on rigid $p$-phenylene units allows the distance to be varied between the $\mathrm{Ru}^{\mathrm{II}}$ metal center and the $\mathrm{TiO}_{2}$ surface from $8 \AA$ (no bridge unit), to $13 \AA$ (one $p$-phenylene bridge unit), and $18 \AA$ (two $p$-phenylene units). The spectra of the excited and oxidized states of $\mathrm{Ru}^{\mathrm{II}}\left(\right.$ terpyridyl- $\left.\mathrm{PO}_{3}\right)(\mathrm{NCS})_{3}$ dyes are easily distinguishable from each other. Hence, by means of ultrafast transient absorption spectroscopy, the characterization of the dynamics of the formation of the oxidized state is simply performed by following the spectral changes that occur upon photoinduced electron injection from the photoexcited state of the dye into the conduction band of the solid oxide, i.e. $\mathrm{S}^{*} \rightarrow \mathrm{S}^{+}+\mathrm{e}_{\mathrm{cb}}{ }^{-}\left(\mathrm{TiO}_{2}\right)$. The obtained PET rate constants were $k_{i n j}=$ $1.2 \cdot 10^{12} \mathrm{~s}^{-1}, 5.9 \cdot 10^{11} \mathrm{~s}^{-1}$, and $2.7 \cdot 10^{11} \mathrm{~s}^{-1}$ for the three sensitizers containing 0,1 and $2 p$-phenylene bridge units, respectively. In this case, the PET distance for charge injection can be represented by the spatial separation between the $\pi^{*}$ donor orbital of the terpyridyl ligand and the nearest acceptor $\mathrm{Ti}^{4+}$ ion of the oxide surface. It varies from $6.5 \AA$ to $15.5 \AA$ for the three compounds. The damping fac- 


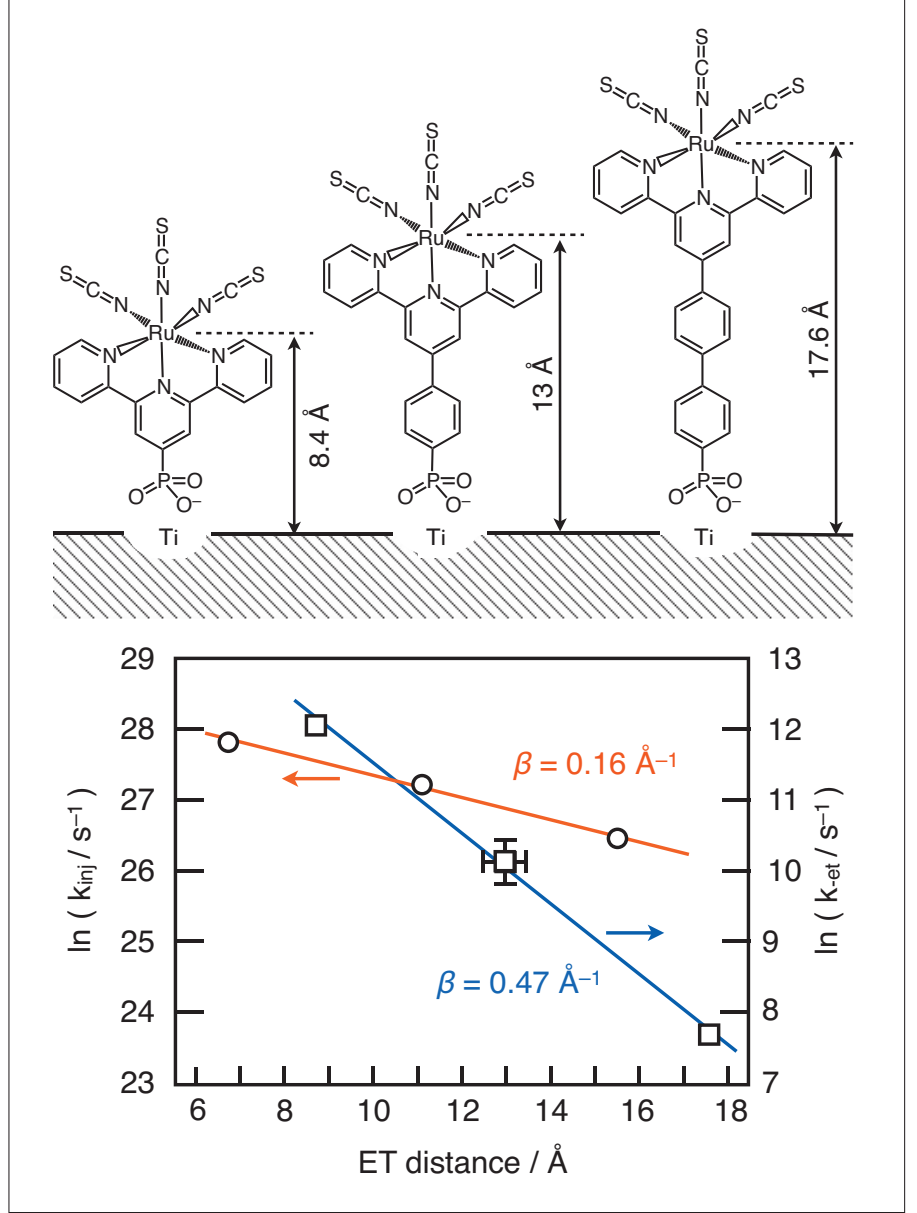

Fig. 3. (Top) Molecular structure of three phosphonated Ru"-terpyridyl complexes equipped with 0,1 and $2 p$-phenylene bridge units separating the chromophore from the phosphonate group anchor. Ru" $\left[2,2^{\prime}: 6^{\prime}, 2^{\prime \prime}-\right.$ terpyridine-4'-phosphonate(NCS), Ru"[2,2':6',2"-terpyridine-4'-phenyl4"-phosphonate(NCS), and Ru"[2,2':6',2"-terpyridine-4'-diphenyl-4"phosphonate(NCS) $)_{3}$ adsorbed on the surface of $\mathrm{TiO}_{2}$ constitute donorbridge-acceptor (D-B-A) systems of increasing D-A distance. (Bottom) Semi-logarithmic plot of the rate constants for forward- $\left(k_{\text {in }}\right)$ and back electron transfer $\left(k_{-e t}\right)$ as a function of the distance separating the donor and the acceptor. The slopes of the observed linear dependence yield damping factor values of $\beta=0.16 \pm 0.03 \AA^{-1}$ and $0.47 \pm 0.05 \AA^{-1}$ for charge injection and recombination processes, respectively.

tor $\beta$ of Eqn. (6) can be estimated from the slope of the semi-logarithmic plot of the rate constant $k_{\text {in, }}$ as a function of the ET distance. Fig. 3 (bottom) shows that electron injection rate constant follows a linear trend, whose slope yields a value of $\beta=0.16 \pm 0.03 \AA^{-1}$.

The kinetics of the recombination reaction between the injected conduction band electrons in titanium dioxide and the oxidized dye species $\mathrm{S}^{+}$at the surface of the nanoparticles i.e. $\mathrm{S}^{+}+\mathrm{e}_{\mathrm{cb}}{ }^{-}\left(\mathrm{TiO}_{2}\right) \rightarrow \mathrm{S}$ was studied by ns transient absorption spectroscopy. The recombination rate of recapturing the electron is observed to be generally slower by several orders of magnitude compared to charge injection rates of efficient sensitizers. For a majority of dye sensitizer/TiO 2 systems, this back electron transfer process typically occurs in the hundreds of $\mu$ s to ms time scale, $10^{6}-10^{10}$ times slower than the initial photoinduced charge injection. ${ }^{[1-3]} \mathrm{At}$ least two main reasons have been invoked to explain this huge difference: (a) Charge recombination takes place between discrete energy levels and is mediated by fluctuation of vibration energy. Thus the rate is scaled down by nuclear factors, which do not intervene in the case of the ultrafast forward electron transfer process. (b) While electron injection is kinetically near optimum, the high exoergicity of the back electron transfer can make the system lie deep in the inverted Marcus region, where the rate of the charge transfer process is expected to decrease with increasing driving force. Fig. 3 displays the dependence of the back electron transfer rate constants, $k_{-e t}$, as a function of ET distance defined as the spatial separation of the $\mathrm{TiO}_{2}$ surface to the acceptor $\mathrm{Ru}^{3+}$ center. Again, an ex- ponential relation was found and a damping factor as obtained from the slope of the semi-logarithmic plot could be estimated to $\beta=0.47 \pm 0.05 \AA^{-1}$. This value is in good agreement with typical damping factors i.e. $\beta=0.5 \pm 0.1 \AA^{-1}$ previously reported for both forward- and back electron transfer in homogeneous oligo- $p$-phenylene bridged donor-acceptor systems. ${ }^{[32]}$ The very different $\beta$ value observed here for charge injection points towards an important decrease of the tunneling energy gap for the forward ET compared to previously reported systems.

The metal-to-ligand charge transfer (MLCT) transition induced by the femtosecond laser pulse at a wavelength of 530 $\mathrm{nm}$ and subsequent ultrafast intersystem crossing to the triplet excited state involves the promotion of an electron from the $\mathrm{Ru}^{\mathrm{II}}$ centered $\mathrm{d}$ orbital to the $\pi^{*}$ orbital of the terpyridine ligand with an excess energy of about $0.3 \mathrm{eV}$. This energy excess absorbed by the donor is deposited in the vibrational modes coupled to the photoinduced electronic transition. As a consequence, this generates a population of nonequilibrium vibrations in the terpyridine ligand, whose lifetime has been established to be as long as 12 ps in solution. Contrary to the ET dynamics measured in other cases where the rate constants did not exceed $10^{10} \mathrm{~s}^{-1}$, electron injection from all three Ru-terpyridyl dyes (PET time constants $\tau_{\text {inj }}=800 \mathrm{fs}-3 \mathrm{ps}$ ) are clearly faster than vibrational relaxation of the hot ${ }^{3}$ MLCT state of the excited complex. It is therefore safe to assume that the exceptionally high electron transmission through the oligo- $p$ phenylene moiety in the excited state of the dye is due to the cooperative coupling of electron tunneling from the terpyridyl ligand to the $\mathrm{TiO}_{2}$ surface with non-equilibrium vibrations redistributed onto the bridge. As a result, the transport of such a non-equilibrium polaron across the interface induces a decrease of the energy gap for electron tunneling and subsequently lowers the damping factor $\beta{ }^{[33]}$

\section{Acknowledgments}

Our research efforts are funded by the Swiss National Science Foundation, through several grants, the National Competence Center for Research NCCR-MUST (Molecular Ultrafast Science and Technology), the Ecole Polytechnique Fédérale de Lausanne, and the European Commission through a Marie Curie project.

Received: July 14, 2011

[1] M. Grätzel, J.-E. Moser, in 'Electron Transfer in Chemistry', Vol. 5, Eds. V. Balzani, I. R. Gould, Wiley-VCH, Weinheim, 2001, p. 589.

[2] J.-E. Moser, in 'Dye-sensitized Solar Cells', Ed. K. Kalyanasundaram, EPFL Press, Lausanne, 2010, Chapter 11, p. 403.

[3] V. K. Thorsmølle, B. Wenger, J. Teuscher, C. Bauer, J.-E. Moser, Chimia 2007, 61, 631. 
[4] J. C. Brauer, V. K. Thorsmølle, J.-E. Moser, Chimia 2009, 63, 189.

[5] A. Punzi, J. C. Brauer, A. Marchioro, E. Ghadiri, J. De Jonghe, J.-E. Moser, Chimia 2011, 65, 353.

[6] V. K. Thorsmølle, G. Rothenberger, D. Topgaard, J. C. Brauer, D.-B. Kuang, S. M. Zakeeruddin, B. Lindman, M. Grätzel, J.E. Moser, ChemPhysChem 2011, 12, 145 .

[7] J. C. Brauer, J. Teuscher, A. Punzi, J.-E Moser, in 'Ultrafast Phenomena XII', Oxford University Press, New York, 2011, p. 358.

[8] a) H. Kisch, W. Lindner, Chemie in unserer Zeit 2001, 35, 250; b) D. Ravelli, D. Dondi, M. Fagnoni, A. Albini, Chem. Soc. Rev. 2009, 38 1999; c) K. Maeda, K. Domen, J. Phys. Chem. Lett. 2010, 1, 2655.

[9] I. R. Gould, J. R. Lenhard, A. A. Muenter, S. A Godleski, S. Farid, Pure Appl. Chem. 2001, 73, 455.

[10] a) M. Grätzel, Nature 2003, 421, 586; b) A. Hagfeldt, G. Boschloo, L. Sun, L. Kloo, H. Pettersson, Chem. Rev. 2010, 110, 6595.

[11] J. Y. Pan, U. Scherf, A. Schreiber, Synth. Met 2000, 115, 79 .

[12] B. Korzeniewski, J. A. Zoladz, Biophys. Chem. 2001, 92, 17 .

[13] C. Aubert, M. H. Vos, P. Mathias, A. P. Eker, K. Brettel, Nature 2000, 407, 926.

[14] J.-D. Rochaix, Biochim. Biophys. Acta 2011, 1807,375 .

[15] R. E. Blankenship, Da. M. Tiede, J. Barber, G. W. Brudvig, G. Fleming, M. Ghirardi, M. R Gunner, W. Junge, D. M. Kramer, A. Melis, T. A. Moore, C. C. Moser, D. G. Nocera, A. J. Nozik, D. R. Ort, W. W. Parson, R. C. Prince, R. T. Sayre, Science 2011, 332, 805.

[16] N. S. Lewis, J. Electroanal. Chem. 2001, 508, 1.

[17] S. M. Sze, K. K. Ng, 'Physics of Semiconductor Devices', Wiley, New York, 2007.

[18] O. Vigil-Galán, E. Marín, J-Sastré Hernández, E. Saucedo, C. M. Ruiz, G. Contreras-Puente, A. Calderón, J. Mater. Sci. 2007, 42, 7176.

[19] a) G. Yu, J. Gao, J. C. Hummelen, F. Wudl, A. J. Heeger, Science 1995, 270, 1789; b) J. J. M. Halls, C. A. Walsh, N. C. Greenham, E. A. Marseglia, R. H. Friend, S. C. Moratti, A. B. Holmes, Nature 1995, 376, 498; c) H. Tamura J. G. S. Ramon, E. R. Bittner, I. Burghardt, Phys. Rev. Lett. 2008, 100, 107402; d) R. Steim, F. R. Kogler, C. J. Brabec, J. Mater. Chem. 2010, 20, 2499.

[20] J. E. Moser, P. Bonhôte, M. Grätzel, Coord. Chem. Rev. 1998, 171, 245.
[21] a) R. Huber, J. E. Moser, M. Grätzel, J. Wachtveitl, J. Phys. Chem. B 2002, 106, 6494; b) R. Huber, J. E. Moser, M. Grätzel, J. Wachtveitl, Proc. SPIE 2003, 5223, 121; c) R. Huber, J. E. Moser, M. Grätzel \& J. Wachtveitl, Chem. Phys. 2002, 285, 39.

[22] Y. Tachibana, J. E. Moser, M. Grätzel, D. R. Klug, J. R. Durrant, J. Phys. Chem. 1996, 100, 20056.

[23] a) S. Pelet, M. Grätzel, J. E. Moser, J. Phys. Chem. B 2003, 107, 3215; b) B. Wenger, M. Grätzel, J. E. Moser, J. Am. Chem. Soc. 2005, 127, 12150

[24] a) R. A. Marcus, Angew. Chem. Int. Ed. 1993, 32, 1111; b) W. R. Duncan, O. V. Prezhdo, Annu. Rev. Phys. Chem. 2007, 58, 143.

[25] a) J. E. Moser, M. Grätzel, Chimia 1998, 52, 160; b) J. E. Moser, M. Wolf, F. Lenzmann, M Grätzel, Z. Phys. Chem. 1999, 212, 85.

[26] a) C. Zimmermann, F. Willig, S. Ramakrishna, B. Burfeindt, B. Pettinger, R. Eichberger, W. Storck, J. Phys. Chem. B 2001, 105, 9245; b) S. Ramakrishna, F. Willig, V. May, J. Chem. Phys. 2001, 115, 2743.

[27] J. M. Lanzafame, S. Palese, D. Wang, R. J. D. Miller, A. A. Muenter, J. Phys. Chem. 1994, 98, 11020.

[28] H. Gray, J. R. Winkler, Proc. Natl. Acad. Sci. USA 2005, 102, 3534 .

[29] a) J. M. Rehm, G. L. McLendon, Y. Nagasawa, K. Yoshihara, J. Moser, M. Grätzel, J. Phys. Chem. 1996, 100, 9577; b) J. Wachtveitl, R. Huber, S. Spörlein, J. E. Moser, M. Grätzel, Int. J. Photoenerg. 1999, 1, 153; c) D. Stockwell, Y. Yang, J. Huang, C. Anfuso, Z. Huang, T. Lian, J. Phys. Chem. C, 2010, 114, 6560.

[30] R. Huber, S. Spörlein, J.-E. Moser, M. Grätzel, J. Wachtweitl, J. Phys. Chem. B 2000, 104, 8995.

[31] C. Bauer, J. Teuscher, S. Pelet, B. Wenger, P. Bonhôte, M. K. Nazeeruddin, S. M. Zakeeruddin, P. Comte, M. Grätzel, J.-E. Moser, Curr. Sci. 2010, 99, 343.

[32] a) A. Helms, D. Heiler, G. McLendon, J. Am. Chem. Soc. 1992, 114, 6227; b) E. A. Weiss, M. J. Ahrens, L. E. Sinks, A. V. Gusev, M. A. Ratner, M. R. Wasielewski, J. Am. Chem. Soc. 2004, 126, 5577; c) M. T. Indelli, C. Claudio, L. Flamigni, L. De Cola, F. Scandola, Inorg. Chem. 2007, 46, 5630.

[33] a) H. Ness, A. J. Fisher, Phys. Rev. Lett. 1999, 83,452 ; b) H. Ness, S. A. Shevlin, A. J. Fisher, Phys. Rev. B, 2001, 63, 125422. 